\title{
Lettre éditoriale
}

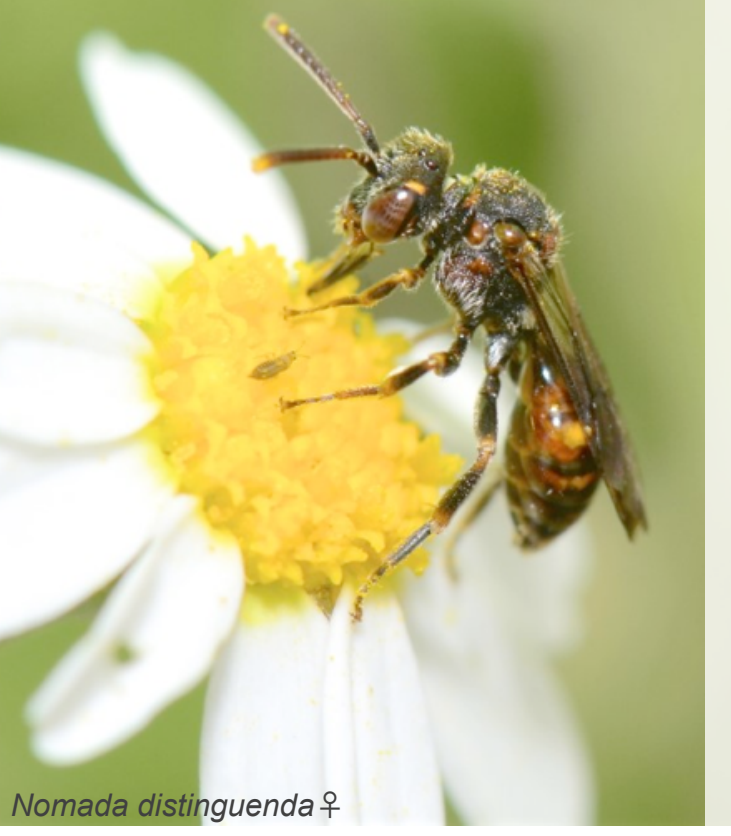

Nomada distinguenda우

\begin{tabular}{|l|}
\hline \multicolumn{1}{|c|}{ COMITÉ } \\
ÉDITORIAL \\
Editeur en chef \\
Benoît Geslin \\
Comité de relecture \\
Matthieu Aubert \\
Éric Dufrêne \\
David Genoud \\
Benoît Geslin \\
Tanguy Jean \\
Gérard Le Goff \\
Violette Le Féon \\
Léa Lemaire \\
Gilles Mahé \\
Denis Michez \\
Géraud de Prémorel \\
Nicolas J.Vereecken \\
\hline
\end{tabular}

\section{SOUMISSION D'ARTICLES}

\section{De l'importance des sciences naturalistes}

"If we do not know where bee species live, and how abundant they are, it is almost impossible to measure decline and generate prioritised and meaningful conservation strategies. » Brown \& Paxton 2009!

Pour beaucoup d'entre nous, cette phrase publiée dans la revue scientifique Apidologie il y a maintenant presque 10 ans raisonne comme un mantra, une profession de foi et elle est plus que jamais d'actualité. II est en effet tout à fait frappant de réaliser à quel point nous manquons encore de connaissances de base sur les abeilles sauvages malgré toutes les études, publications et autres analyses qui paraissent chaque année. Pour une partie non négligeable d'entre elles, nous ignorons encore tout simplement leur répartition, leur écologie et la dynamique de leur population. Dans ce cadre, le développement des connaissances naturalistes sur les Apoidés sauvages - une des aspirations de l'Observatoire des Abeilles - reste un objectif majeur qui doit tendre à se développer dans les années futures.

e nouveau numéro d'Osmia est une illustration parfaite de l'importance des sciences naturalistes pour la progression des connaissances sur les abeilles. En effet, ce ne sont pas moins de 3 espèces nouvelles pour la faune de France métropolitaine qui sont présentées dans ce septième numéro. Tout d'abord, E. Gabiot et E. Dufrêne font pour la première fois mention de Nomada numida dans l'hexagone. Une autre espèce du genre, Nomada rubricoxa, y a également été découverte récemment dans le cadre d'une étude menée dans le Parc National des Calanques (Geslin et al.). Enfin, Ghisbain et al. étoffent le genre Dasypoda en y ajoutant une neuvième espèce à savoir D. morawitzi. Ce numéro d'Osmia s'attache aussi à accroître les connaissances sur les communautés d'abeilles sauvages dans les milieux anthropisés (Paris, Ropars et al.) ou semi-naturels (Parc National des Calanques, Geslin et al.). Enfin, les aires de répartition nationale telles que connues à ce jour d'un de nos bourdons les plus majestueux, Bombus argillaceus, et d'une espèce exotique envahissante, Megachile sculptularis, font l'objet d'une présentation détaillée, respectivement par B. Frin ainsi que par V. Le Féon et al. Nous inaugurons également une nouvelle rubrique dans la partie Forum dans ce numéro puisque T.Jean présente ici ses conseils de lecture pour tous les passionnés de taxonomie et d'écologie des abeilles sauvages. Pour finir, vous y retrouverez une magnifique planche photo réalisée par $G$ de Premorel.

Nous espérons que vous apprécierez ce numéro autant que nous avons eu plaisir à le rédiger .... et que vivent les sciences naturalistes !

Benoît GESLIN, Editeur d'Osmia, pour le comité de rédaction. 